\title{
Design of polymeric materials for culturing human pluripotent stem cells: progress toward feeder-free and xeno-free culturing
}

\begin{abstract}
This review describes recent developments regarding the use of natural and synthetic polymers to support the propagation of human pluripotent stem cells (hPSCs), human embryonic stem cells (hESCs), and induced pluripotent stem cells (hiPSCs) while maintaining pluripotency in feeder-free and xeno-free cultures. The development of methods for culturing these cells without using mouse embryonic fibroblasts (MEFs) as a feeder layer will enable more reproducible culture conditions and reduce the risk of xenogenic contaminants, thus increasing the potential clinical applications of differentiated hPSCs. Human or recombinant fibronectin, laminin-511, and vitronectin, which are components of the extracellular matrix (ECM), have been used instead of Matrigel for the feeder-free growth of undifferentiated hPSCs. Successful hPSC cultures have been described for the following conditions: on oligopeptide-immobilized surfaces derived from vitronectin, on microcarriers prepared from synthetic polymers, and encapsulated within three-dimensional (3D) hydrogels composed of alginate and other hydrophilic natural polymers. Recently, synthetic biomaterials that allow hPSCs to maintain pluripotency by secreting endogenous ECM components have been designed. The combination of human ECM proteins or cell adhesion molecules (e.g., oligopeptides and poly-d-lysine) and synthetic biomaterials with welldesigned surfaces and/or structures (e.g., scaffolds, hydrogels, microcarriers, microcapsules, or microfibers) in the presence of a chemically defined medium containing recombinant growth factors would offer a xeno-free alternative to feeder cells for culturing hPSCs and maintaining their pluripotency.
\end{abstract}

Keyword: Biomaterial; Embryonic stem cells; Extracellular matrix; Cell culture; Hydrogel; Microcarrier; Microcapsule 